\title{
Measuring and Controlling Unfairness in Decentralized Planning of Energy Demand
}

\author{
Evangelos Pournaras ${ }^{\# 1}$, Matteo Vasirani ${ }^{* 2}$, Robert E. Kooij !\#3, Karl Aberer *4 \\ \# Department of Computer Science and Engineering \\ Delft University of Technology \\ Delft, The Netherlands \\ ${ }^{1}$ e.pournarasdtudelft.nl \\ ${ }^{3}$ r.e.kooijetudelft.nl \\ * Distributed Information Systems Laboratory \\ EPFL \\ Lausanne, Switzerland \\ 2 matteo.vasiranidepfl.ch \\ ${ }^{4}$ karl-aberer@epfl.ch \\ ! TNO \\ Delft, The Netherlands \\ ${ }^{3}$ robert.kooijetno.nl
}

\begin{abstract}
Demand-side energy management improves robustness and efficiency in Smart Grids. Load-adjustment and loadshifting are performed to match demand to available supply. These operations come at a discomfort cost for consumers as their lifestyle is influenced when they adjust or shift in time their demand. Performance of demand-side energy management mainly concerns how robustness is maximized or discomfort is minimized. However, measuring and controlling the distribution of discomfort as perceived between different consumers provides an enriched notion of fairness in demand-side energy management that is missing in current approaches. This paper defines unfairness in demand-side energy management and shows how unfairness is measurable and controllable by software agents that plan energy demand in a decentralized fashion. Experimental evaluation using real demand and survey data from two operational Smart Grid projects confirms these findings.
\end{abstract}

Index Terms-unfairness, fairness, agent, planning, demand, Smart Grid, load-adjustment, load-shifting.

\section{INTRODUCTION}

Demand-side energy management can make Smart Grids more robust and efficient as demand-response mechanisms are capable of preventing black-outs, increasing utilization of renewable energy resources, reducing carbon emissions or decreasing the electricity prices for consumers. Demandresponse mechanisms are mainly designed to maximize the load-adjustment or load-shifting potential. However, these mechanisms do not usually take into consideration the impact that demand-side energy management has on the lifestyle of consumers. Load-adjustment and load-shifting are likely to cause discomfort, which might be not equally distributed between consumers. This effect makes most demand-side energy management approaches unfair, and such unfairness may result in a lower adoption level of demand-response programs or even in malfunctioning of electricity markets [1] and monetary compensations [2].
This paper studies unfairness in decentralized planning of energy demand. Unfairness is defined by the dispersion of discomfort that consumers experience when future demand is planned in response to a load-adjustment or load-shifting event. Different consumers perceive discomfort in a different way. For example, a room with low temperature does not make all consumers feel the same cold. This paper shows how reasoning about the discomfort perception of consumers is possible by analyzing the answers that consumers provided in surveys of demand-response programs. This contribution provides a more realistic view of unfairness that is closer to Smart Grid practice.

This paper also shows that unfairness is temporally influenced and correlated to the demand level, which varies seasonally. For example, in geographic areas that have cold winters with higher demand than summers, unfairness is higher. This paper also shows how unfairness is locally controllable by tuning the process of demand planning. If the number of alternative demand plans is lower in load-adjustment, unfairness is also lower. This paper experimentally studies the aforementioned aspects using real demand data from two operational Smart Grid projects. Given that fairness (or unfairness) is a relatively unexplored research area in demand-side energy management, this paper shows that unfairness is measurable and controllable in Smart Grids, concluding that a notion of fairness can provide a more in-depth understanding about the performance trade-offs in demand-side energy management.

This paper is outlined as follows: Section II introduces the context of demand planning in which unfairness is studied. Section III motivates and defines a notion of unfairness in demand planning. Section IV illustrates two operational Smart grid projects from which data are used for the experimental evaluation that follows in Section V. Section VI illustrates and compares the notion of unfairness introduced in this paper with 
related work. Finally, Section VII concludes this paper.

\section{Demand Planning}

Demand planning is defined in this paper as the computation of a time series, the demand plan, with the scheduled energy consumption of a future time period. This paper studies demand planning at the household level, yet, this approach can be extended to the level of a household appliance, wall outlet or even the feeder of a neighborhood. Demand planning can be used as a computational mechanism of demand-side energy management services under various demand-response programs [3]. This paper focuses on two such services that can be used to improve the robustness and efficiency of Smart Grids: (i) load-adjustment and (ii) load-shifting.

Load-adjustment in demand planning is defined as an overall increase or decrease of demand compared to an intended demand without planning. A decrease in demand is required, for example, if the overall capacity of supply is not adequate to meet demand. An increase in demand is desired when there is a high availability of renewable energy resources [4] or when market prices are low [5]. In practice, load-adjustment is achieved by incentive mechanisms [6] that motivate changing the comfort level of heating/cooling or switching on/off household appliances.

Load-shifting in demand planning is defined as a change in demand distribution compared to the one of an intended demand without planning, yet the overall average planned demand equals the intended one. Load-shifting often results in peak-shaving, minimization of power oscillations or shifting of load to low-peak hours. In practice, load-shifting is achieved by pre-heating/cooling water heaters before peak-hours or incentivizing consumers to change their consumption behavior, e.g. making a shower earlier or later in time.

This paper focuses on the planning model of possible demand plans [7] that are locally generated by software agents (or other software components) that control a household energy management system [8]. The possible plans represent alternative demand plans from which an agent can choose, according to local criteria and system objectives.

Plan selections can be local or coordinated. This paper focuses on load-adjustment objectives under local selections: demand is minimized by letting agents choose demand plans with the lowest average energy consumption. This can be achieved with a fitness function that locally minimizes energy demand. Thus, coordination between different agents is not required. In contrast, load-shifting objectives require coordinated selections that result in desynchronizing the times during which consumers consume energy. Such a coordination is performed by EPOS, the Energy Plan Overlay Self-stabilization system [9].

This paper adopts a data-driven approach for plan generation. Possible demand plans are locally generated by clustering historical demand data every certain time period, e.g., every day, for a total period of time, e.g., a week or a month. Clustering is based on the computation of a proximity metric such as the Euclidean or the Manhattan distance [10]. The number of clusters is usually part of the clustering parameterization and represents the number of possible plans that the agents generate. The total period of time from which historic data is used as input of the clustering algorithm can be defined by a sliding clustering window. For example, the CAISO demand forecasting methodology predicts demand based on the energy consumption of the past 10 days [11]. The same principle can be adopted for the generation of the possible plans for the following day.

Each possible plan is devised by computing the representative demand time series of each cluster. More specifically, each possible plan is the medoid of a cluster and is computed by the median of the historical time series that belongs to this cluster. A critical aspect in the clustering process is the number of clusters $l$ that corresponds to the number of possible plans. Previous experimental work shows that a higher number of possible plans in demand-side energy management results in improved robustness for Smart Grids [9], [7]. A higher number of possible plans means that the agents have a higher degree of freedom to adjust demand according to system objectives. However, a higher number of possible plans increases also the computational $\operatorname{cost}^{1}$ and causes a lower cluster size on average. A cluster with a lower size results in a devised possible plan that is less representative of past energy consumption.

\section{UNFAIRNESS UNDER DEMAND PLANNING}

Planning of demand for load-adjustment and load-shifting results in a discomfort impact for consumers. Changes are required that affect how and when consumers use energy. For example, load-adjustment may entail that heating temperature setpoints are lower during winter times resulting in a discomfort experience: consumers feel colder. The same holds for load-shifting as consumers need to change the times when they perform certain activities, e.g. running the washing machine. Moreover, a discomfort experience depends on human perception and therefore the discomfort impact of load-adjustment and load-shifting is not the same among different consumers.

Most demand-side energy management methods do not consider how this discomfort is distributed among consumers. The distribution of discomfort indicates a degree of fairness in the sense of how 'equally' consumers contribute to the robustness of Smart Grids. This paper introduces a method that quantifies and controls unfairness of discomfort under load-adjustment and load-shifting, even when consumers perceive discomfort differently. Two types of discomfort are distinguished: (i) adjustment discomfort and (ii) shifting discomfort.

Adjustment discomfort is assumed to be the discomfort experienced under load-adjustment and it is defined by summing positive and negative errors that correspond to the distance of the planed demand from the actual demand. Adjustment discomfort is computed as follows:

\footnotetext{
${ }^{1}$ The increased computational cost concerns the generation process but also the optimization performed by EPOS [9].
} 


$$
A_{i}=w_{i}^{a} \sum_{t=1}^{T}\left(p_{i}^{t}-d_{i}^{t}\right),
$$

where $p_{i}^{t}$ is the demand at time $t$ of the selected plan with size $T$ generated and selected by agent $i$. The demand $d_{i}^{t}$ is the respective intended energy consumption without demand planning. Negative errors are perceived as negative discomfort, or comfort, given that consumers use a higher amount of energy resources. The weight $w_{i}^{a} \in[0,1]$ represents the sensitivity of a consumer $i$ to the adjustment discomfort. A value equals to 0 means that the consumer does not perceive any adjustment discomfort due to demand reduction, while a value equal to 1 means that the sensitivity of the consumer to demand reduction is maximal.

Shifting discomfort is assumed as the discomfort experienced under load-shifting and it is defined by the root mean square deviation as follows:

$$
S_{i}=w_{i}^{s} \sqrt{\frac{1}{T} \sum_{t=1}^{T}\left(p_{i}^{t}-d_{i}^{t}\right)^{2}},
$$

where $p_{i}^{t}$ and $d_{i}^{t}$ are the same demand values defined for $A_{i}$. In contrast to adjustment discomfort, shifting discomfort measures squared errors that are always positive and capture temporal changes in demand. As before, the weight $w_{i}^{s} \in[0,1]$ represents the sensitivity of consumer $i$ to any shifting of the desired demand $d_{i}^{t}$ : the higher the value of $w_{i}^{s}$, the higher the perceived discomfort.

The discomfort values between $n$ different consumers cannot be compared as they correspond to different demand levels. For this reason, normalized discomfort values in the range $[0,1]$ are computed as follows:

$$
\begin{aligned}
A_{i}^{\prime} & =\frac{A_{i}-\min _{i}^{n}\left(A_{i}\right)}{\max _{i}^{n}\left(A_{i}\right)-\min _{i}^{n}\left(A_{i}\right)}, \\
S_{i}^{\prime} & =\frac{S_{i}-\min _{i}^{n}\left(S_{i}\right)}{\max _{i}^{n}\left(S_{i}\right)-\min _{i}^{n}\left(S_{i}\right)}
\end{aligned}
$$

Finally, the unfairness values $U_{A}$ and $U_{S}$ are quantified by computing the standard deviation of the normalized adjustment and shifting discomfort values:

$$
\begin{aligned}
U_{A} & =\sqrt{\frac{1}{n} \sum_{i=1}^{n}\left(A_{i}^{\prime}-{\overline{A^{\prime}}}_{i}\right)}, \\
U_{S} & =\sqrt{\frac{1}{n} \sum_{i=1}^{n}\left(S_{i}^{\prime}-{\overline{S^{\prime}}}_{i}\right)}
\end{aligned}
$$

where ${\overline{A^{\prime}}}_{i}$ and ${\overline{S^{\prime}}}_{i}$ are the mean of all adjustment and shifting discomfort values.

Although the notion of fairness is relevant to other aspects of demand-side energy management, e.g., monetary compensation, this paper focuses on the novel aspect of discomfort that is challenging to quantify. Moreover, if discomfort is fairly distributed among consumers, this prevents situations in which consumers may aim for themselves the full monetary benefit [2].

\section{VAlidation in SMART GRID PROJECTS}

The actual unfairness that consumers experience via demand planning is validated a posteriori using real consumption data from two operational Smart Grid projects, the Electricity Customer Behavior Trial ${ }^{2}$ in Ireland and the Olympic Peninsula Smart Grid Demonstration ${ }^{3}$ in the USA. The intended energy demand $d_{i}^{t}$ used to compute the discomfort values is referred to in this paper as CONTROL-DATA.

\section{A. The Electricity Customer Behavior Trial project}

This project is a cost-benefit analysis that assesses the impact of different time-of-use tariffs on the electricity consumption of Irish households and enterprises. The project ran in the period 2009-2010 with 5000 residential and business consumers participating. The data are cleaned from missing values and filtered out to contain the energy consumption time series of 782 residential consumers that belong to the control group ${ }^{4}$.

The software agent that controls the energy management system reasons about the number of possible plans based on the answers provided by the human consumer to the following two survey questions ${ }^{5}$ :

Question 1. My household may decide to make minor changes to the way we use electricity.

Question 2. My household may decide to make major changes to the way we use electricity.

The answer $a_{q}$ in each of the above question $q$ belongs to $\{1, \ldots, 5\}$, where 1 stands for a strong agreement and 5 stands for a strong disagreement. Algorithm 1 illustrates how agents reason about the number of possible plans they generate ${ }^{6}$. Note that the number of plans computed by this algorithm is referred to in this paper as $l=f_{1}(z=x)$. The main intuition behind the generation algorithm is the normalization of the answers to the two questions $a_{1}, a_{2}$ in $l \in\{z-2, \ldots, z+3\}$. The number of possible plans increases as consumers allow for more major changes in the way they use electricity (lines 1-6 in Algorithm 1). The constant $z$ is used as a scaling factor for the number of possible plans in the generation process.

\footnotetext{
${ }^{2}$ http://www.ucd.ie/issda/data/commissionforenergyregulationcer/ (last accessed January 2014)

${ }^{3}$ https://svn.pnl.gov/olypen/ (last accessed January 2014)

${ }^{4}$ These consumers are not affected by the time-of-use tariffs applied for the purpose of the project.

${ }^{5}$ The question block ' 55122 ' of the pre-trial residential survey contains these two questions.

${ }^{6}$ Note that from the total number of 782 residential consumers, 132 of these do not participate in the pre-trial survey. For 116 of these consumers, the question block '54132' of the post-trial survey is used for computing Algorithm 1. This question block is the respective post-trial question block '55122' of the pre-trial survey (My household made minor/major changes to the way we use electricity.). For the final 16 residential consumers that do not participate in neither of the pre-trial nor post-trial surveys, the number of possible plans is computed by the median number of possible plans in the the rest of the 766 consumers.
} 

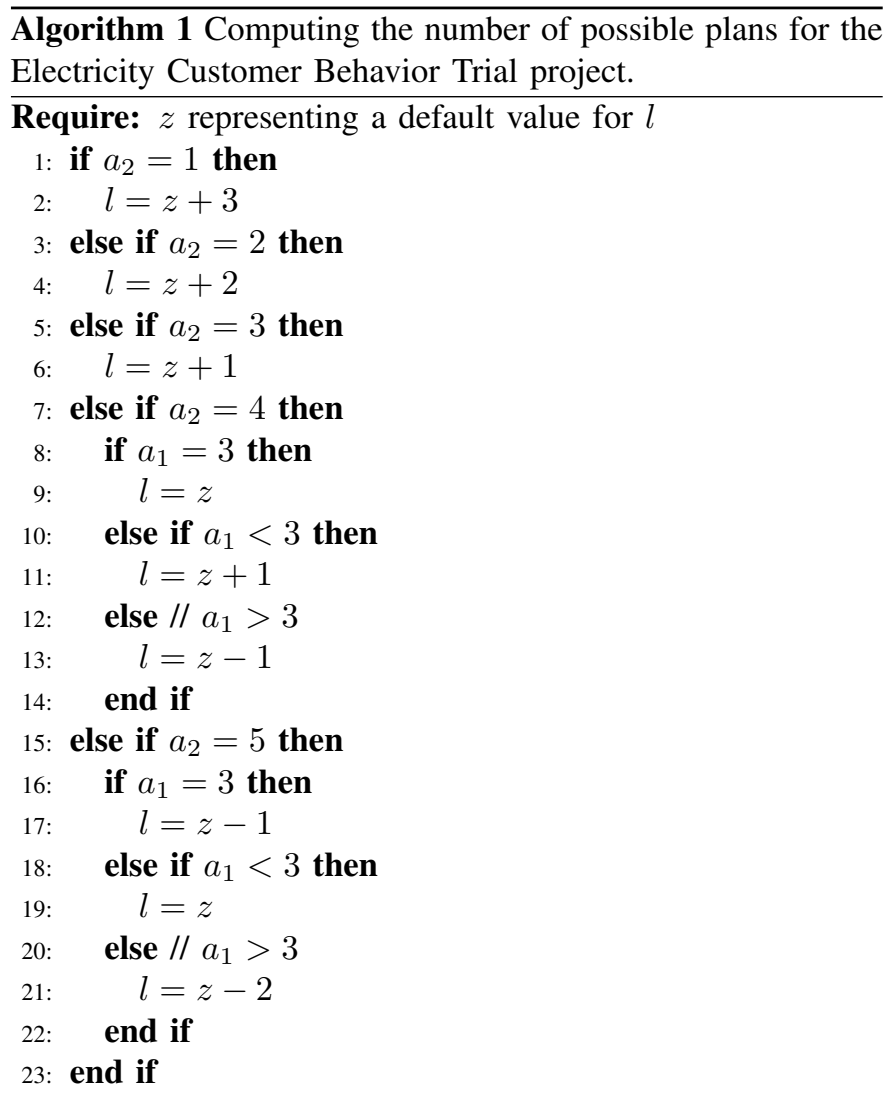

Ensure: number of possible plans $l$ for each agent

The weights of discomfort are computed by the answers of consumers to the following two questions:

Question 3. It is too inconvenient to reduce our usage of electricity ${ }^{7}$.

Question 4. I am interested in changing the way I use electricity if it helps environment ${ }^{8}$.

Based on the possible answers $\{1, \ldots, 5\}$, where 1 stands for a strong agreement and 5 stands for a strong disagreement, the weights $w_{i}^{s}$ and $w_{i}^{a}$ for each agent $i$ are computed by normalizing the answers in the range $[0,1]$.

\section{B. The Olympic Peninsula Smart Grid Demonstration project}

This project assesses the adjustment of individual energy usage based on price signals exchanged within a two-way bidding market [12]. The project concerns the period of March 2006 to March 2007 with 112 household participants regionally distributed in the Olympic Peninsula of the USA. The data subset from November 2006 to March 2007 is selected during which the lowest number of missing values is observed. The demand of each consumer is captured every 5 minutes. Demand data are aligned to the sampling rate of the Electricity Customer Behavior Trial project by aggregating

\footnotetext{
${ }^{7}$ This is question ' 4352 ' in the residential pre-trial survey.

${ }^{8}$ This is question ' 4331 ' in the residential pre-trial survey.
}

12 consecutive demand bids of each hour to a single hourly demand bid.

Demand data are filtered out to contain 29 consumers that (i) either belong to the CONTROL group or have a FIXED type of contract and (ii) have less than $20 \%$ of their values missing. Two extra consumers are excluded as their demand time series contains a large proportion of zero values. Therefore the final number of consumers used in the evaluation is 27 . The missing values in the final consumers are interpolated by computing the average demand values in the past and future 10 days.

In the context of this project, the demand adjustment is achieved by dynamically modifying the temperature setpoints of various household devices. Motivated by this approach, the number of possible plans $l=f_{2}(z=x)$ is defined by a function that captures the selected temperature setpoints of consumers during project runtime. More specifically, the range of minimum and maximum temperature setpoints selected is normalized to $l \in\{z, \ldots, z+4\}$ for a given constant $z$.

\section{EXPERIMENTAL EVALUATION}

This section experimentally evaluates unfairness under demand planning in the two Smart Grids projects illustrated in Section IV. It also shows how a global metric such as unfairness can be locally controlled by the number of plans that agents generate.

The possible plans available to the agents are generated by clustering the energy consumption time series of the 10 most recent days that is the length of the sliding clustering window. The number of plans is selected according to two different criteria: (i) by assigning a default number of plans to each agent and (ii) by computing the number of possible plans based on project data. In the first case, the minimum number of $l=2$ is selected. This number minimizes the computational cost in each agent ${ }^{9}$. In the second case, the agents reason about the consumer preferences collected by each project, e.g., survey answers and temperature setpoints. Load-adjustment and load-shifting are performed on a random day of each week, simulating in this way a demand-response event. An implementation of the hierarchical clustering algorithm [13] in Weka $^{10}$ is used for generating the possible plans ${ }^{11}$ of the agents with the value of $z=2$. Figure 1 illustrates the normalized histogram of the number of possible plans $l$ in the two Smart Grid projects. Figure 2 illustrates the normalized histogram of the two discomfort weights, computed from the answers of consumers in Question 3 and 4.

Given that load-shifting requires coordinated plan selections as illustrated in Section II, an implementation of EPOS in the Protopeer [14] toolkit is used for this purpose. EPOS coordinates demand by letting agents interact over self-organized tree topologies [15]. For the same tree topology, 10 instances are generated, in each of which the agents are positioned

\footnotetext{
${ }^{9}$ Unfairness is also minimized as shown in this paper.

${ }^{10} \mathrm{http}: / / w w w . c s . w a i k a t o . a c . n z / m l / w e k a /$ (last accessed September 2013)

${ }^{11}$ Note that if $l \leq 1$, then an agent selects the median time series from the sliding clustering window
} 


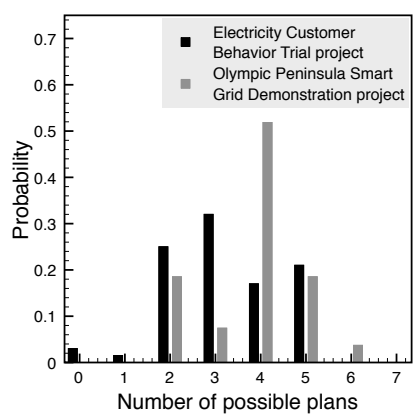

Fig. 1. The normalized histogram for the number of possible plans $l$ under $z=2$.

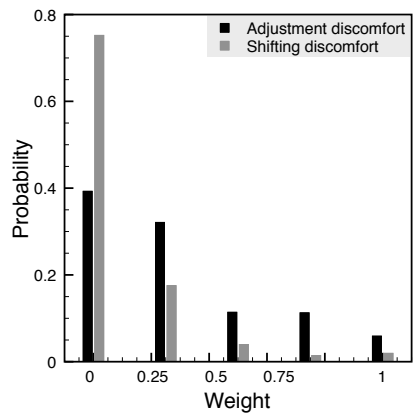

Fig. 2. The normalized histogram of shifting $w_{i}^{s}$ and adjustment $w_{i}^{a}$ dis comfort weights derived from the pre-trial survey of the Electricity Customer Behavior Trial project.

randomly, so as to eliminate possible bias introduced by a certain topological positioning of the agents.

\section{A. Temporal Influence}

Figure 3 illustrates unfairness when load-adjustment is performed. For the Electricity Customer Behavior Trial project in Figure 3a, a temporal influence is observed. During winter time, unfairness increases, while during summer time (July and August), unfairness is minimal. Figure $4 b$ does not show a clear temporal influence. A possible explanation is that for this Smart Grid project a significantly lower number of consumers is available, whose energy consumption data spans over a shorter time range (from November to March) that does not reach summer time.

Figure 4 illustrates unfairness when load-shifting is performed. The same temporal pattern is observed in Figure 4a as in Figure 3a. Unfairness in demand planning is higher in winter time than in summer time. Figure $4 \mathrm{~b}$ shows lower unfairness in November than in the rest of the winter days.

In order to understand these temporal patterns, the total demand from Control-Data for the two Smart grid projects is plotted in Figure 5. The total demand of Irish households is higher in winter than in summer. The same holds for the households of Olympic Peninsula that reach the highest demand on December and January.

Note that there is a strong correlation between the unfairness and the total demand for both Smart Grid projects as

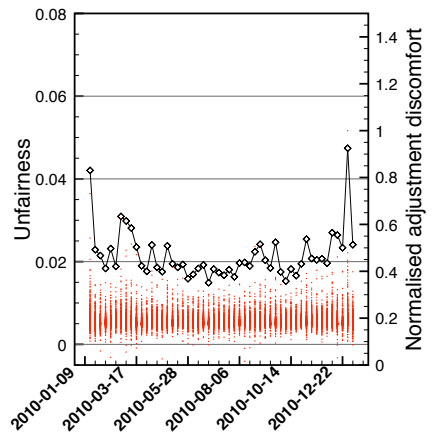

(a) Electricity Customer Behavior Trial project.

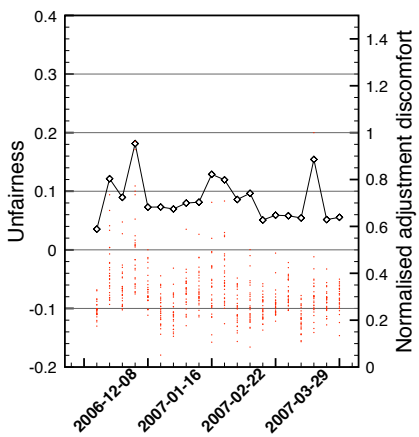

(b) Olympic Peninsula Smart Grid Demonstration project.
Fig. 3. The values of adjustment discomfort under load-adjustment for each agent (dots with values on the right $\mathrm{Y}$ axis). Their dispersion shows the unfairness for the two Smart Grid projects (line with values on the left Y axis).

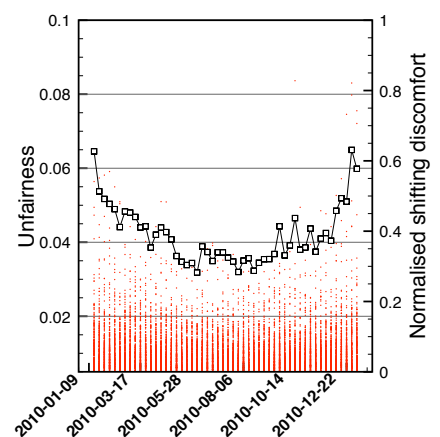

(a) Electricity Customer Behavior Trial project.

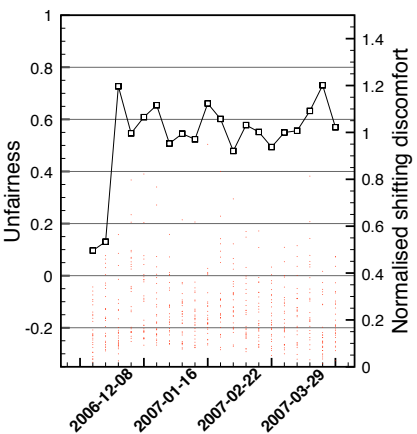

(b) Olympic Peninsula Smart Grid Demonstration project.
Fig. 4. The values of shifting discomfort under load-shifting for each agent (dots with values on the right $\mathrm{Y}$ axis). Their dispersion shows the unfairness for the two Smart Grid projects (line with values on the left $\mathrm{Y}$ axis).

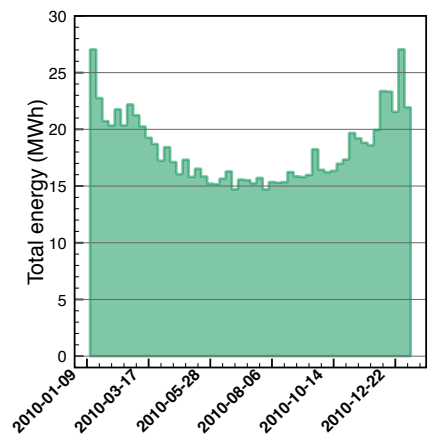

(a) Electricity Customer Behavior Trial project.

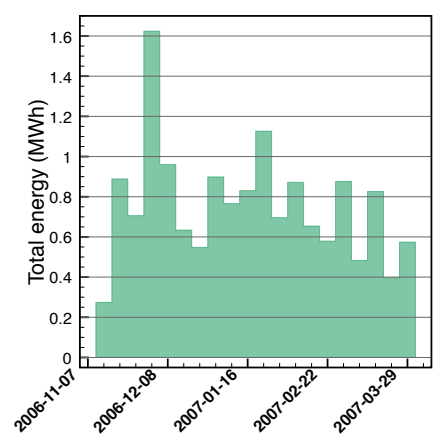

(b) Olympic Peninsula Smart Grid Demonstration project.
Fig. 5. The total demand from Control-DatA for the two Smart Grid projects.

illustrated in Table I. The Pearson correlation coefficient is always positive for both load-adjustment and load-shifting. For example, the correlation coefficient is 0.92 between unfairness (Figure 4a) and the total demand (Figure 5a) under loadshifting. The low but positive correlation of 0.15 under loadshifting in the Olympic Peninsula Smart Grid Demonstration 
project is explained by the low number of consumers that participate in demand planning.

TABLE I

THE CORRELATION COEFFICIENTS BETWEEN UNFAIRNESS AND THE TOTAL DEMAND FROM CONTROL-DATA.

\begin{tabular}{|l|c|c|}
\cline { 2 - 3 } \multicolumn{1}{c|}{} & Load-adjustment & Load-shifting \\
\hline Electricity Customer Behavior Trial & 0.80 & 0.92 \\
\hline $\begin{array}{l}\text { Olympic Peninsula Smart Grid } \\
\text { Demonstration }\end{array}$ & 0.82 & 0.15 \\
\hline
\end{tabular}

The positive correlation values show that a temporal influence of unfairness comes from the fact that weather influences the demand level of consumers. If demand is higher under load-adjustment and load-shifting, a higher unfairness becomes more probable in the discomfort that consumers experience.

\section{B. Control of Unfairness}

Albeit unfairness is a global metric, it can be locally controlled by influencing the number of possible plans that the agents generate. This section studies the effect that the number of possible plans have in unfairness. For this purpose, agents are grouped according to the number of plans they generate as shown in Figure 1. Given that the values of unfairness are normalized as shown in Equation (3) and (4), the two groups are compared in Figure 6 and 7 under load-adjustment. In both figures, unfairness increases as the number of plans increases.

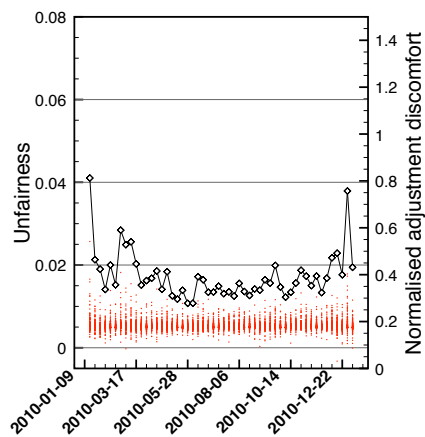

(a) $l=2$.

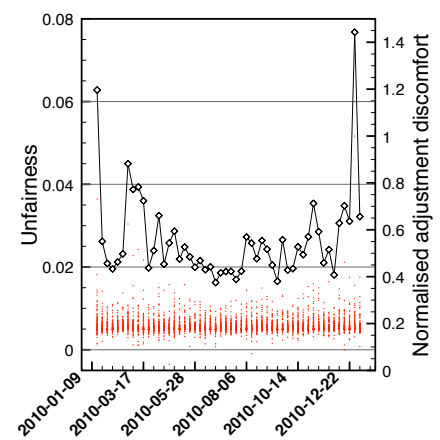

(b) $l=4$
Fig. 6. The values of adjustment discomfort under load-adjustment for agents with different number of possible plans $l$ (dots with values on the right $\mathrm{Y}$ axis). Their dispersion shows the unfairness for the Electricity Customer Behavior Trial project (line with values on the left $\mathrm{Y}$ axis).

More specifically, unfairness increases $53.4 \%$ on average when the number of possible plans increases from 2 to 4 for the Electricity Customer Behavior Trial project. The respective increase is $39.0 \%$ on average for the Olympic Peninsula Smart Grid Demonstration project.

The unfairness for different number of possible plans under load-shifting is $2.9 \%$ higher on average for the Electricity Customer Behavior Trial project. This increase does not indicate a clear influence as it is significantly lower than the one in load-adjustment.

Unfairness is not the only factor that is influenced by the number of possible plans that agents generate. Earlier work shows that a higher number of possible plans increases the

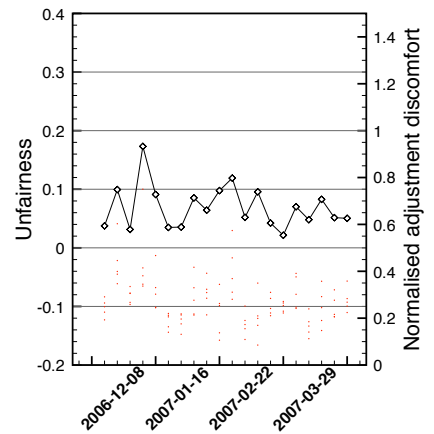

(a) $l=2$.

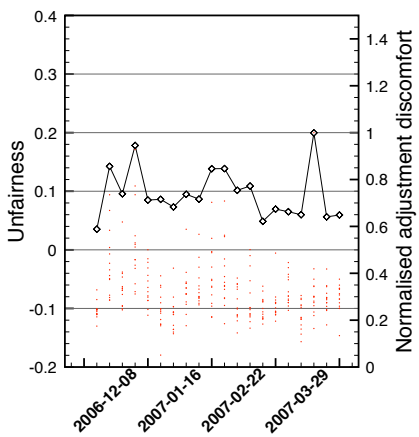

(b) $l=4$.
Fig. 7. The values of adjustment discomfort under load-adjustment for agents with different number of possible plans $l$ (dots with values on the right $\mathrm{Y}$ axis). Their dispersion shows the unfairness for the Olympic Peninsula Smart Grid Demonstration project (line with values on the left $\mathrm{Y}$ axis).

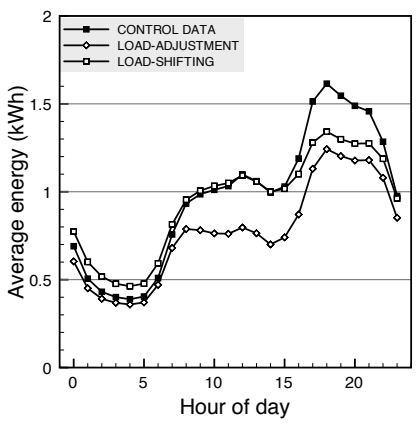

(a) Electricity Customer Behavior Trial project.

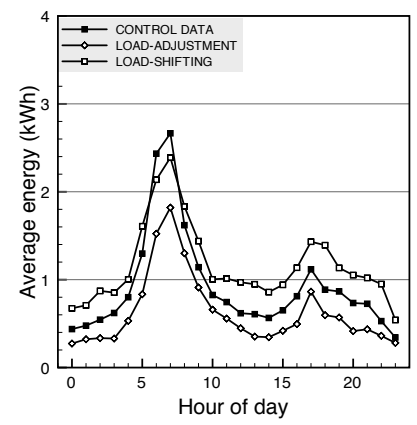

(b) Olympic Peninsula Smart Grid Demonstration project.
Fig. 8. Average daily energy demand for the two Smart Grid projects.

potential of stabilizing energy consumption, since the agents have a higher degree of freedom to make choices that match system-wide objectives [7]. Figure 9 and 10 show the different demand curves achieved in groups of consumers whose agents generate different number of possible plans. Figure 8 shows the respective curves for the total number of agents.

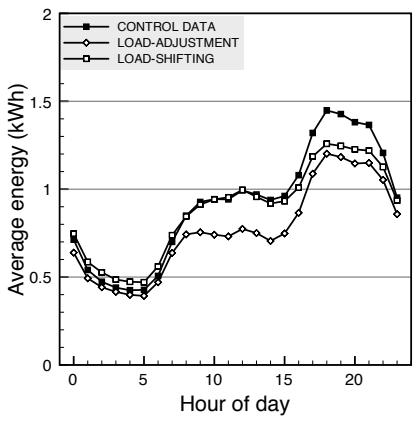

(a) $l=2$.

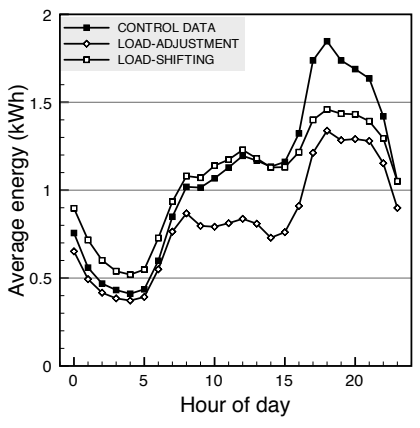

(b) $l=4$.
Fig. 9. Average daily energy demand of agents with different number of possible plans $l$ for the Electricity Customer Behavior Trial project.

The average daily demand curves show that as the number of possible plans increases, the load-adjustment and load-shifting demand curves differ to a higher extent from 
CONTROL-DATA. In practice, the morning and evening power peaks under load-adjustment experience a more significant reduction with $l=4$ than with $l=2$ in both Smart Grid projects. Under load-shifting, a higher demand increase is observed during low-peak times as $l$ increases, for example, the early morning times in both Smart Grid projects.

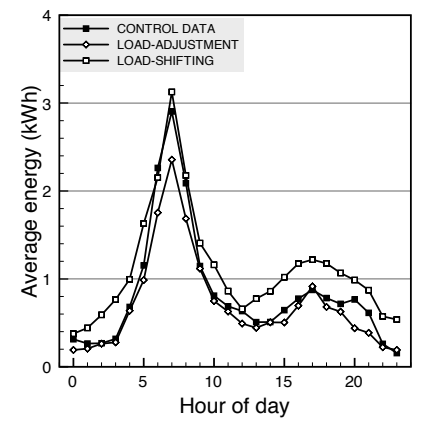

(a) $l=2$.

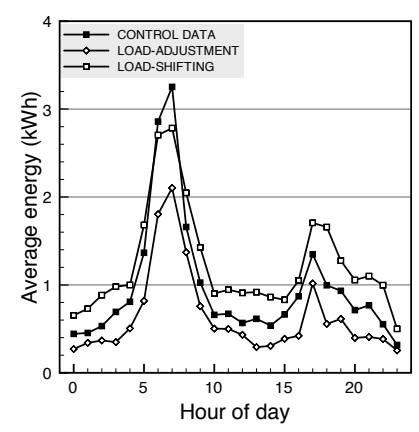

(b) $l=4$.
Fig. 10. Average daily energy demand of agents with different number of possible plans $l$ for the Olympic Peninsula Smart Grid Demonstration project.

These demand curves indicate that a more effective loadadjustment and load-shifting is ensured by consumers with a higher number of possible plans, who also experience a higher discomfort, causing in this way higher unfairness at a global level.

\section{Summary of findings}

The following findings summarize the experimental evaluation of this section:

1) Unfairness in demand planning is temporally influenced. For example, unfairness is higher in winter than in summer.

2) The temporal influence of unfairness in demand planning is correlated to the different demand levels observed during the year.

3) Unfairness in demand planning is locally controllable by the number of possible plans that the agents generate: the lower the number of possible plans, the lower the unfairness.

4) A more effective load-adjustment and load-shifting results in a higher unfairness in demand-planning.

\section{COMPARISON With RELATED WORK}

Fairness (or unfairness) in demand-side energy management is a relatively unexplored research area. This section outlines the most relevant work that is related to this paper.

Stadler et al. argue that communication protocols are a key aspect of a market-based demand-side ancillary service to ensure fairness [1]. If, for example, a request for load shedding is small enough to be fulfilled by a single participant in the ancillary service market, it might be that the fastest participant to retrieve the request always wins the monetary incentive attached to it. With a well-designed communication protocol, load shedding requests can be delivered to all the participants at the same time, or alternatively in a round-robin fashion, to ensure that all participants have their turn [3].

It is shown that billing mechanisms can be designed to be fair in autonomous demand-response mechanisms [2]. Fairness is achieved by rewarding consumers according to their contributions to meet system-wide objectives. In contrast to this approach, the concept of unfairness introduced in this paper focuses on how discomfort is distributed among consumers. Moreover, the authors of [2] do not focus on the factors that affect fairness and how fairness can be controlled by the system designer.

A fair allocation of power to air-conditioners is introduced in [16]. Two notions of fairness are studied, (i) the min-max fairness that concerns the lowest temperature that consumers can have and (ii) the proportional fairness that deals with the fraction of requested cooling that each consumer has. Fairness is controlled in a centralized fashion by utility companies, in contrast to this work that introduces a local control of fairness by adjusting the number of possible plans that agents generate.

A similar centralized optimization approach for fairness and discomfort is introduced by [17], in which two scheduling algorithms are proposed: (i) highest power next and (ii) round robin. Discomfort is monitored according to the duration in which room temperatures deviate from the desired temperature, whereas this paper evaluates discomfort a posteriori, using real datasets from two operational Smart Grid projects.

\section{CONCLUSION}

This paper concludes that unfairness in the distribution of discomfort between consumers is measurable and controllable in decentralized planning of energy demand. This paper shows how reasoning about the perception of consumers in discomfort is possible. It also shows that unfairness is temporally influenced given the correlation of unfairness with different seasonal demand levels observed in real demand data. When load-adjustment and load-shifting become more effective, unfairness increases. This observation shows a performance trade-off in the design of demand-response programs that provides a more in-depth understanding about the role of consumers in demand-side energy management. The findings of this paper can be used to improve and motivate a broader adoption of fair demand-respond programs in Smart Grids.

\section{ACKNOWLEDGMENT}

This work is funded by the NWO project RobuSmart (grant agreement No. 647.000.001), the FP7 European projects Wattalyst (grant agreement No. 288322) and EINS (grant agreement No. 288021). Authors would like to thank Tri Kurniawan Wijaya for his software support on clustering. Authors are also grateful to the Irish Social Science Data Archive and the Pacific Northwest National Laboratory for providing access to power demand data.

\section{REFERENCES}

[1] M. Stadler, P. Palensky, B. Lorenz, M. Weihs, and C. Roesener, "Integral resource optimization networks and their techno-economic constraints," International Journal on Distributed Energy Systems, vol. 1, no. 4, pp. 299-320, 2005. 
[2] Z. Baharlouei, M. Hashemi, H. Narimani, and H. Mohsenian-Rad, "Achieving optimality and fairness in autonomous demand response: Benchmarks and billing mechanisms," Smart Grid, IEEE Transactions on, vol. 4, no. 2, pp. 968-975, 2013.

[3] P. Palensky and D. Dietrich, "Demand Side Management: Demand Response, Intelligent Energy Systems, and Smart Loads," IEEE Transactions on Industrial Informatics, vol. 7, no. 3, pp. 381-388, Aug. 2011.

[4] C. Brandstätt, G. Brunekreeft, and K. Jahnke, "How to deal with negative power price spikes? Flexible voluntary curtailment agreements for largescale integration of wind," Energy Policy, vol. 39, no. 6, pp. 3732-3740, Jun. 2011

[5] P. Cappers, C. Goldman, and D. Kathan, "Demand response in U.S electricity markets: Empirical evidence,” Energy, vol. 35, no. 4, pp. 1526-1535, Apr. 2010

[6] P. Joskow and J. Tirole, "Reliability and Competitive Electricity Markets," The RAND Journal of Economics, vol. 38, no. 1, pp. 60-84, May 2007.

[7] E. Pournaras, "Multi-level reconfigurable self-organization in overlay services," Ph.D. dissertation, Delft University of Technology, March 2013.

[8] A. Kailas, V. Cecchi, and A. Mukherjee, "A Survey of Communications and Networking Technologies for Energy Management in Buildings and Home Automation," Journal of Computer Networks and Communications, vol. 2012, pp. 1-12, Dec. 2012.

[9] E. Pournaras, M. Warnier, and F. M. T. Brazier, "Local Agent-based Self-stabilisation in Global Resource Utilisation," International Journal of Autonomic Computing, vol. 1, no. 4, pp. 350 - 373, Dec. 2010.

[10] R. Giancarlo, G. Lo Bosco, and L. Pinello, "Distance functions, clustering algorithms and microarray data analysis," in Learning and Intelligent Optimization, ser. Lecture Notes in Computer Science, C. Blum and R. Battiti, Eds. Springer Berlin Heidelberg, 2010, vol. 6073, pp. 125138.

[11] A. Sfetsos and C. Siriopoulos, "Time series forecasting of averaged data with efficient use of information," Systems, Man and Cybernetics, Part A: Systems and Humans, IEEE Transactions on, vol. 35, no. 5, pp. 738$745,2005$.

[12] D. J. Hammerstrom, "Part I. Olympic Peninsula Project," Pacific Northwest National Laboratory, Richland, WA, USA, Tech. Rep., Oct. 2007

[13] A. K. Jain, "Data clustering: 50 years beyond k-means," Pattern Recognition Letters, vol. 31, no. 8, pp. 651 - 666, 2010.

[14] W. Galuba, K. Aberer, Z. Despotovic, and W. Kellerer, "ProtoPeer: A P2P Toolkit Bridging the Gap Between Simulation and Live Deployement," in Proceedings of the Second International Conference on Simulation Tools and Techniques, ICST 2009. Gent, Belgium: ACM, Mar. 2009, pp. 1-9.

[15] E. Pournaras, M. Warnier, and F. M. T. Brazier, "Adaptation Strategies for Self-management of Tree Overlay Networks," in Proceedings of the 11th IEEE/ACM International Conference on Grid Computing, Grid 2010. Los Alamitos, CA, USA: IEEE, Oct. 2010, pp. 401-409.

[16] Y.-W. Chen, X. Chen, and N. Maxemchuk, "The fair allocation of power to air conditioners on a smart grid," Smart Grid, IEEE Transactions on vol. 3, no. 4, pp. 2188-2195, 2012.

[17] G. Koutitas, "Control of flexible smart devices in the smart grid," Smart Grid, IEEE Transactions on, vol. 3, no. 3, pp. 1333-1343, 2012. 